\title{
BMJ Open Vitamin D in the prevention of exacerbations of asthma in preschoolers (DIVA): protocol for a multicentre randomised placebo-controlled triple- blind trial
}

\author{
Megan E Jensen, ${ }^{1}$ Francine M Ducharme, ${ }^{2,3,4}$ Nathalie Alos, ${ }^{2,5}$ \\ Geneviève Mailhot (D) , ${ }^{6}$ Benoît Mâsse, ${ }^{7,8}$ John H White, ${ }^{9}$ Mohsen Sadatsafavi, ${ }^{10}$ \\ Ali Khamessan, ${ }^{11}$ Sze Man Tse, ${ }^{2,3}$ Reza Alizadehfar, ${ }^{12}$ Dirk E Bock, ${ }^{13}$ \\ Patrick Daigneault, ${ }^{14}$ Chantal Lemire, ${ }^{15}$ Connie Yang, ${ }^{16}$ Dhenuka Radhakrishnan ${ }^{17}$
}

To cite: Jensen ME

Ducharme FM, Alos N, et al. Vitamin $\mathrm{D}$ in the prevention of exacerbations of asthma in preschoolers (DIVA): protocol for a multicentre randomised placebo-controlled triple-blind trial. BMJ Open 2019;9:e033075. doi:10.1136/ bmjopen-2019-033075

- Prepublication history and additional material for this paper are available online. To view these files, please visit the journal online (http://dx.doi. org/10.1136/bmjopen-2019033075).

Received 19 July 2019 Revised 16 0ctober 2019 Accepted 23 October 2019

D Check for updates

(C) Author(s) (or their employer(s)) 2019. Re-use permitted under CC BY-NC. No commercial re-use. See rights and permissions. Published by BMJ.

For numbered affiliations see end of article.

\section{Correspondence to}

Francine M Ducharme; francine.m.ducharme@ umontreal.ca

\section{ABSTRACT}

Introduction Preschoolers have the highest rate of emergency visits and hospitalisations for asthma exacerbations of all age groups, with most triggered by upper respiratory tract infections (URTIs) and occurring in the fall or winter. Vitamin D insufficiency is highly prevalent in Canadian preschoolers with recurrent asthma exacerbations, particularly in winter. It is associated with more URTIs and, in patients with asthma, more oral corticosteroid (OCS) use. Although evidence suggests that vitamin D supplements significantly decrease URTIs and asthma exacerbations requiring OCS, there is insufficient data in preschoolers. This study aims to determine the impact of vitamin $\mathrm{D}_{3}$ supplementation on exacerbations requiring OCS, in preschoolers with recurrent URTI-induced asthma exacerbations.

Methods and analysis This is a phase III, randomised, triple-blind, placebo-controlled, parallel-group multicentre trial of vitamin $D_{3}$ supplementation in children aged 1-5 years, with asthma triggered by URTIs and a recent history of frequent URTIs and OCS use. Children $(n=865)$ will be recruited in the fall and early winter and followed for 7 months. They will be randomised to either the (1) intervention: two oral boluses of 100000 international unit (IU) vitamin $\mathrm{D}_{3}$ (3.5 months apart) with $400 \mathrm{IU}$ vitamin $\mathrm{D}_{3}$ daily; or (2) control: identical placebo boluses with daily placebo. The primary outcome is the number of exacerbations requiring OCS per child, documented by medical and pharmacy records. Secondary outcomes include number of laboratory-confirmed viral URTIs, exacerbation duration and severity, parent functional status, healthcare use, treatment deintensification, cost and safety.

Ethics and dissemination This study has received ethical approval from all sites. Results will be disseminated via international conferences and manuscripts targeting paediatricians and respirologists, and to families of asthmatic children via our Quebec parents-partners outreach programme. If proven effective, findings may markedly influence the management of URTI-induced asthma in high-morbidity preschoolers and could be
Strengths and limitations of this study

- This multicentre randomised controlled trial will be the largest paediatric study testing the impact of high-dose vitamin D supplementation, compared with placebo, as adjunct to inhaled corticosteroids, in preschool-aged children with predominantly viralinduced asthma.

- This trial uses a pragmatic patient selection and easily applicable intervention to maximise subsequent implementation in practice.

- The main outcome, the number of exacerbations treated with rescue oral corticosteroids, was selected by parents-partners as clinically meaningful, which can be successfully ascertained by pharmacy and medical records in all randomised participants: it is likely to change practice if a $25 \%$ reduction is documented.

Because of the variability in diet, vitamin D supplement use, sun exposure and skin colour, it is impossible to control all factors that may affect circulating 25-hydroxyvitamin D levels; however, it is expected that these factors will be balanced between groups due to randomisation and recruitment only in fall and early winter.

- Despite the ethical requirement of allowing eligibility for children taking up to 400 IU of supplemental vitamin D, there is a low risk of dilution of effect, given that the average dietary and supplemental intake in the target population is $60 \%$ lower than recommended.

directly implemented into practice with an update to clinical guidelines.

Trial registration number NCT03365687.

\section{INTRODUCTION}

Preschool-aged children have the highest rate of emergency department (ED) visits 
and hospitalisations due to wheeze/asthma among all age groups, ${ }^{1}$ with annual ED visit rates of $23-42 / 1000^{2}$; on average, one in three are hospitalised following an ED visit. ${ }^{2}$ Most exacerbations are triggered by viral upper respiratory tract infections (URTIs), ${ }^{3} 4$ which occur frequently in preschool-aged children. URTIs cause more severe exacerbations than other triggers, ${ }^{5}$ and are associated with poorer response to oral corticosteroids (OCS) ${ }^{6}$ Although daily or pre-emptive high-dose inhaled corticosteroids (ICS) as monotherapy have been identified as effective management strategies for preschool asthma, ${ }^{7}$ $50 \%$ still require rescue OCS within 6 months. ${ }^{8-10}$ Yet, in case of treatment failure, there is no official recommendation for step-up therapy as there is only one published trial in preschool-aged children testing the benefit of adjunct therapy to ICS. ${ }^{11}$ The dearth in knowledge to manage high-morbidity preschoolers with ongoing exacerbations despite ICS monotherapy underlines the crucial need for new prevention strategies. ${ }^{12}$

A 2016 systematic review of randomised controlled trials (RCT) testing vitamin D supplementation in patients with asthma found vitamin $\mathrm{D}$ reduced the rate of exacerbations requiring OCS by $36 \%$ (RR $0.64,658$ adults/22 preschoolers) and acute-care visits/hospitalisations by $61 \%$ (OR 0.39, 664 adults/277 school-aged children/22 preschoolers). ${ }^{13}$ Due to the under-representation of preschoolers and participants with moderate/severe asthma, considerable heterogeneity in vitamin $\mathrm{D}$ dosing and ICS cointervention, and the paucity of trials, there was insufficient power to explore subgroup differences; it thus remains unclear whether patient (eg, age, baseline serum 25-hydroxyvitamin D (25OHD) level, genetic variation) or treatment (eg, vitamin D schedule, ICS use) characteristics modify the magnitude of benefit. Subsequent paediatric trials using various populations, interventions and outcomes led to conflicting results. ${ }^{1415}$ Clearly, there is a need for an adequately powered, confirmatory efficacy trial, to determine if vitamin $\mathrm{D}_{3}$ supplementation, as adjunct to ICS, reduces exacerbations requiring OCS in preschoolers with viral-induced asthma, irrespective of serum baseline 25OHD. ${ }^{16}$

Exploration of potential mechanisms of action would further elucidate the role of vitamin $\mathrm{D}$ in asthma. A protective effect of vitamin D on upper (OR 0.64$)^{17}$ and lower (OR 0.58$)^{18}$ respiratory infections, and in schoolaged children with viral-induced asthma (RR 0.26), ${ }^{19}$ has been previously reported. However, the absence of laboratory confirmation of infection in most trials raises the possibility of outcome misclassification: only one trial objectively documented infection (influenza A), reporting a significant reduction (RR 0.58 ) in exacerbations with vitamin D supplements. ${ }^{20}$ In most tissues, ${ }^{21-23}$ infection or allergen exposure induce the expression of enzyme CYP27B1 that converts 25OHD into active 1,25-dihydroxyvitamin $\mathrm{D}$; the latter binds to nuclear vitamin $\mathrm{D}$ receptor, which regulates the transcription of over 1000 genes, ${ }^{24}{ }^{25}$ including several associated with bronchial smooth muscle cells, ${ }^{26}$ explaining the observed inverse relationship between serum 25OHD and airway reactivity. ${ }^{27}$ An immune-modulating and synergistic effect of vitamin D with ICS on airway inflammation may also exist, with a paediatric RCT reporting a significant reduction in ICS dose in the vitamin D group compared with controls ${ }^{28}$ confirming observational findings ${ }^{29-32}$ While bone health is optimised with serum $25 \mathrm{OHD} \geq 75 \mathrm{nmol} / \mathrm{L}$, that is, vitamin D sufficiency, ${ }^{33}$ the thresholds for optimal immune, anti-inflammation and respiratory function are unknown, but believed to be higher. Hence, exploration of the impact of vitamin D on immune and inflammatory mechanistic pathways ${ }^{34-36}$ would enhance understanding of mechanisms of action.

In contrast to healthy children, ${ }^{37}$ vitamin $\mathrm{D}$ insufficiency is highly prevalent year-round in children with asthma, ${ }^{298-41}$ peaking in winter. ${ }^{42}$ In order to prevent the well-documented September asthma epidemic ${ }^{43}$ associated with URTIs, a rapid increase in, and maintenance of, serum 25OHD over the fall and winter is crucial. A single oral bolus of 100000 international unit (IU) vitamin $\mathrm{D}_{3}$ rapidly increases $25 \mathrm{OHD}$ levels in preschoolers, ${ }^{40}$ but levels are not sustained without a repeat bolus and daily supplementation. In our first pilot RCT, a single bolus of 100000 IU was compared with a single placebo bolus, with both groups receiving daily $400 \mathrm{IU}$ vitamin $\mathrm{D}_{3}$ for 6 months: the intervention significantly raised serum 25 OHD with $100 \%$ versus $55 \%$ of preschool-aged children, respectively, achieving a sustained level $\geq 75 \mathrm{nmol} / \mathrm{L}$ at 3 months. Despite a modest further increase in 25OHD, attributed to daily supplementation, there was no significant group difference at 6 months, underlying the need for a repeat bolus. ${ }^{41}$ In our second pilot RCT, comparing two 100000 IU vitamin $\mathrm{D}_{3}$ versus placebo boluses, 3.5 months apart, without daily supplementation, a rapid increase in serum 25OHD was observed 10 days postbolus; however, serum 25OHD returned to baseline levels, with no significant group difference at 3.5 months, underlying the need for daily supplementation. ${ }^{40}$ This is concordant with prior literature suggesting no sustained effect of bolus supplementation alone on serum 25OHD or health outcomes. ${ }^{4041}{ }^{44-61}$ In our pilot trials, only two participants in the intervention group had a serum $25 \mathrm{OHD}>250 \mathrm{nmol} / \mathrm{L}$ after the first or second bolus; however, this was not associated with hypercalciuria or hypercalcaemia and there were no cases of vitamin D toxicity (serum $25 \mathrm{OHD}>500 \mathrm{nmol} / \mathrm{L}$ ). ${ }^{40}{ }^{41} \mathrm{We}$ concluded that two bolus doses of $100000 \mathrm{IU}$, given 3.5 months apart, with daily supplements of 400 IU vitamin $\mathrm{D}_{3}$ would ensure a rapid and sustained increase of serum 25OHD. Although routinely used in Europe,${ }^{62}$ safety data remain scarce: $:^{45} 4750$ contributing additional safety data on 100000 IU bolus use appears worthwhile.

The protocol of a placebo-controlled parallel-group triple-blind RCT to explore the impact of vitamin $\mathrm{D}_{3}$ supplementation on reducing exacerbations in preschoolers with viral-induced asthma is described herein, as per Standard Protocol Items: Recommendations for Interventional Trials guidelines (online supplementary file 1 ). 


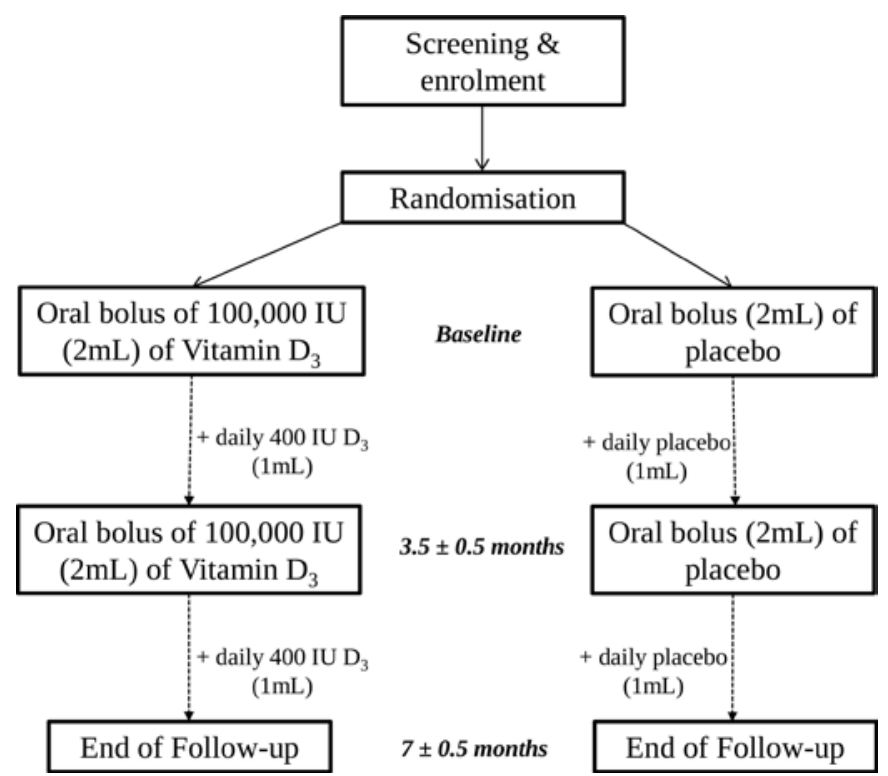

Figure 1 Study design and participant flow through the $7 \pm 0.5$ month study.

The trial has been designed with consideration of several key factors, namely (i) pragmatic enrolment of eligible children irrespective of atopy, phenotype and baseline serum 25OHD to facilitate implementation; (ii) timing of intervention in the fall and early winter, with study period continued throughout winter to coincide with the peak incidence of exacerbations and URTIs and seasonal decline in serum 25OHD; (iii) use of a safe, easily implementable intervention, to achieve a rapid and sustained rise in serum 25OHD, with bolus administration coinciding with usual clinic visits; (iv) ICS prescribed in all participants according to Canadian recommendations ${ }^{63}$; (v) selection of a clinically meaningful, parent-approved primary efficacy outcome known to influence practice; (vi) exploration of potential mechanisms of action and (vii) safety profile documentation. If proven effective, this approach would markedly influence paediatric asthma management and could be directly implemented in clinical practice.

\section{Research questions and study hypothesis Objectives}

The primary question is whether two oral doses of $100000 \mathrm{IU}$ vitamin $\mathrm{D}_{3}$ (administered 3.5 months apart) plus daily $400 \mathrm{IU}$ vitamin $\mathrm{D}_{3}$ decrease the number of asthma exacerbations requiring OCS, versus placebo, in preschool-aged children with recurrent moderate or severe viral-induced exacerbations, treated with daily or pre-emptive high-dose ICS.

Additionally, the study will examine if, compared with placebo, the vitamin D intervention: (i) reduces the mean number of laboratory-confirmed viral respiratory infections; reduces the (ii) severity, (iii) duration of asthma symptoms and (iv) the intensity of rescue $\beta_{2}$-agonist use, during exacerbations; (v) improves parental functional status during exacerbations; (vi) reduces the mean number of asthma-related ED visits; (vii) is associated with de-intensification of ICS therapy and (viii) is costeffective. This study will examine the intervention's safety profile by assessing whether there is a group difference in the proportion of children with: clinically significant (ix) hypercalciuria and (x) hypercalcaemia; (xi) serum $25 \mathrm{OHD}>250 \mathrm{nmol} / \mathrm{L}^{64}$ and (xii) adverse health events (AHE). It will also assess various exploratory outcomes.

\section{Hypothesis}

We hypothesise that vitamin $\mathrm{D}_{3}$ supplementation will reduce the number of exacerbations requiring OCS per child by $25 \%$, versus placebo, in children aged $1-5$ years with recurrent moderate or severe viral-induced asthma exacerbations, and who are not at risk for vitamin D deficiency at baseline.

\section{METHODS AND DESIGNS Study design}

This is a multicentre, phase III, randomised, tripleblind, placebo-controlled, parallel-group trial comparing supplemental vitamin $\mathrm{D}_{3}$ and placebo, both administered as adjunct to ICS (figure 1).

This study is conducted over 4 years (first participant recruited: 1 October 2018; estimated study completion: December 2022) at seven Canadian hospitals: SainteJustine University Hospital Centre (CHUSJ), McGill University Health Centre (MUHC), Sherbrooke University Hospital Centre, Quebec University Hospital Centre, Children's Hospital of Eastern Ontario, Children's Hospital London Health Sciences Centre and British Columbia Children's Hospital. Additional sites will be enlisted, if needed.

\section{Subjects}

Children are eligible if they are aged $1-5$ years, with a physicians' diagnosis of asthma based on clinical signs of airflow obstruction and reversibility according to Canadian guidelines, ${ }^{63}$ a recent history of asthma exacerbation(s) requiring OCS ( $\geq 1$ in the past 6 months or $\geq 2$ in the past year, documented in pharmacy and/or medical records), frequent URTIs ( $\geq 4$ in the past year) and URTIs identified by parents as the main asthma trigger.

Children are excluded if they meet any of the following criteria: current intake/intention to use $>400 \mathrm{IU} /$ day of vitamin D supplement, or combined dietary and supplemental vitamin D intake that would exceed the recommended daily upper limit (ie, 2500 IU for children aged $1-3$ years and 3000 IU for children aged $4-8$ years) ${ }^{65}$ if combined with the intervention dose; extreme prematurity ( $<28$ weeks gestation); no vitamin D supplementation if exclusively breastfed in the past 6 months; vitamin $\mathrm{D}$ restrictive diet; undernourished (body mass index (BMI)-for-age in children $\geq 2$ years of age, or either weight or length for age in those $<2$ years, less than the third percentile); recent (<1 year) refugees and immigrants from regions at high risk of rickets; other chronic 
respiratory disease; diagnosed condition(s) or use of medication(s) that alter calcium or vitamin D absorption/metabolism and anticipated follow-up difficulties. Exclusions minimise the risk of including children with rickets and vitamin $\mathrm{D}$ deficiency without screening for serum 25OHD at baseline.

\section{Study intervention}

Intervention group participants receive a $2 \mathrm{~mL}$ oral bolus of $100000 \mathrm{IU}$ vitamin $\mathrm{D}_{3}$ (50000 IU cholecalciferol/mL) at randomisation in the fall or early winter, followed by a second $2 \mathrm{~mL}$ oral bolus of $100000 \mathrm{IU}$ vitamin $\mathrm{D}_{3} 3.5 \pm 0.5$ months later. Participants also receive a total of five $50 \mathrm{~mL}$ coded bottles, containing a $400 \mathrm{IU}$ vitamin $\mathrm{D}_{3} / \mathrm{mL}$ preparation, to be administered at a dose of $1 \mathrm{~mL} /$ day using a dropper, for $7 \pm 0.5$ months. Each bottle contains 50 daily doses. Placebo group participants receive an identical $2 \mathrm{~mL}$ placebo bolus, and daily dose of $1 \mathrm{~mL}$ placebo preparation, with administration timing identical to the intervention group.

\section{Randomisation and masking}

Using a computer-generated random list, stratified by site, children are allocated in a 1:1 ratio to the intervention or placebo group, using permuted block randomisation method to enhance concealment. Site-specific group allocation codes are held locally in a secure location with restricted access by each Site pharmacy, the CHUSJ Central Pharmacy and the independent biostatistician. The manufacturer (Euro-Pharm International Canada Inc., Montreal, QC Canada) provides the vitamin $\mathrm{D}_{3}$ and placebo preparations, identical in appearance and taste, in coded latex-free bottles. The allocated treatment number, obtained at enrolment through a web-based randomisation system, Research Electronic Data Capture (REDCap) ${ }^{66}$ is forwarded to the Site pharmacy, which prepares the bolus in a coded syringe and the coded daily dose bottles, in masked kits. Research personnel administer orally the coded bolus dose to participants and provide daily dose bottles. Treatment compliance is ascertained by confirmation of bolus retention and pharmacy weighting of returned bottles. All participants, parents, research personnel, physicians and analysts involved in the trial are blinded to group allocation; only pharmacists are unblinded. At study endpoint, parents, physicians and research personnel are asked to guess the child's group allocation. Efficacy and safety analyses will be performed under allocation concealment, with unblinding after trial completion and primary outcome analysis.

\section{Cointerventions}

Following Canadian guidelines, ${ }^{63}$ daily ICS or pre-emptive high-dose ICS with/without additional therapies, as per clinical judgement, is required at randomisation. Where calcium intake is identified as insufficient via dietary recall, dietary change or calcium supplements sufficient to meet the daily calcium Estimated Average Requirement
(500 mg for children 1-3 years; $800 \mathrm{mg}$ for children 4-8 years) is recommended. ${ }^{65}$

\section{Study procedures \\ Screening}

Potentially eligible children are approached and screened for enrolment in asthma, chest or allergy clinics, asthma education centres, EDs and hospital units at participating sites from April to January; a successful strategy in previous trials. ${ }^{84041}$ Advertisements may be placed in health institutions, newspapers, social media and online, where parents are invited to complete an online screening form and, if eligible and interested, to solicit a phone appointment with the research team to confirm eligibility, explain the study and schedule the randomisation visit.

\section{Randomisation and follow-up contact visits}

Randomisation occurs between 1 September and 31 January, with a 7-month follow-up. Participants attend three research visits (randomisation, $3.5 \pm 0.5$ months, and $7 \pm 0.5$ months) and receive six follow-up phone calls ( $7 \pm 3$ days after each bolus, then monthly). Each contact serves to screen for AHEs; inquire about recent exacerbations and URTIs, related parental work absenteeism and expenses, and concomitant medication use; and remind parents to complete questionnaires and collect a nasal swab during events (table 1).

\section{Covariates}

At baseline, skin colour is assessed using a visual adaptation of the validated Fitzpatrick scale. ${ }^{40} 4167$ A blood sample is also drawn for a subsequent analysis of specific serum IgE (Phadiatop + Fx5 food allergen mix or equivalent) ${ }^{68} \mathrm{~A}$ nasal secretion sample is taken at randomisation and $3.5 \pm 0.5$ months using a paediatric flocked mid-turbinate swab, placed in a tube containing $1 \mathrm{~mL}$ Universal Transport Medium (Copan Italia, Brescia, Italy), and stored at $-80^{\circ} \mathrm{C}$ before quantification of the number and type of viruses using PCR analysis (Research St. Joseph's, Hamilton, ON Canada) ${ }^{69} 70$ At each visit, height and weight are measured, and dietary and sun exposure questionnaires, are completed.

\section{During an event}

During a flare-up or URTI, parents are instructed to collect a nasal swab and complete: (1) the validated 17-item Asthma Flare-up Diary for Young Children $(\mathrm{ADYC})^{71}$ daily throughout the event to document symptoms and, at the end of the event, both the (2) validated 21-item Effect of a Child's Asthma flare-up on Parents (ECAP) questionnaire $^{72}$ to measure parental quality of life and (3) summary-of-event form to capture healthcare utilisation, including hospital/pharmacy name. Parent collection of nasal swabs has been shown to be comparable to physician collection. ${ }^{73}$ Nasal swabs are frozen at $-20^{\circ} \mathrm{C}$ until delivered (courier or in person) to the recruitment site and stored at $-80^{\circ} \mathrm{C}$ prior to PCR analysis. Reminders containing instructions with links to complete online 


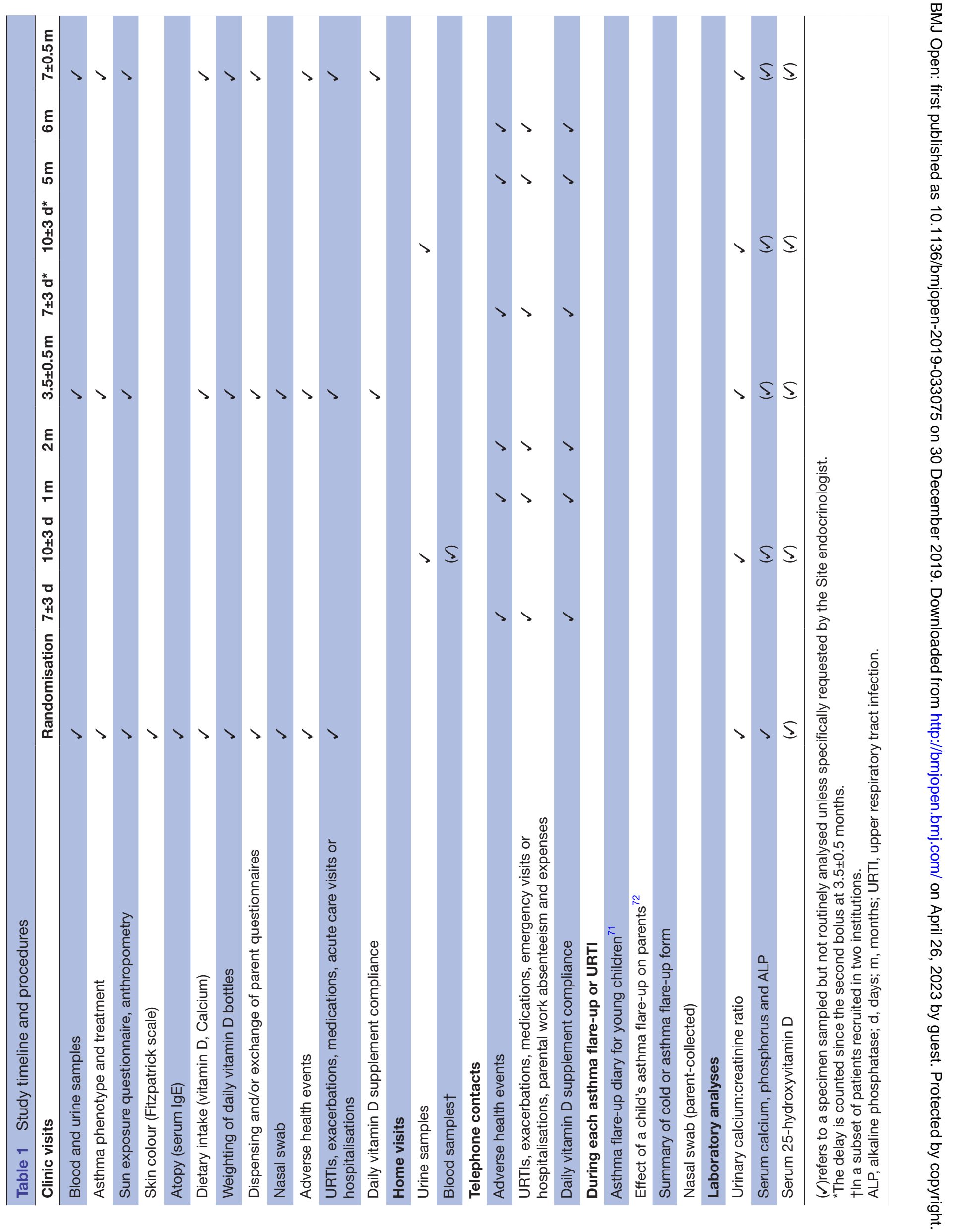


questionnaires are sent by SMS or email at randomisation then regularly throughout the study period.

\section{Risk management}

Clinical and biochemical AHEs are monitored throughout the study and will be reported for all patients at the end of the study. A urine sample is systematically collected and analysed for calcium:creatinine ratio five times, that is, at each visit and at $10 \pm 3$ days following each bolus. A non-fasting venous blood sample is collected at each visit for all participants and, in a subset of consenting participants, of the CHUSJ and MUHC, systematically at $10 \pm 3$ days following the first bolus for peripheral blood mononuclear cell extraction in the two sites (CHUSJ and MUHC) able to process these samples. Blood samples are obtained for four main purposes: (i) individual patient's safety assessment (eg, serum calcium (Ca), phosphorus $(\mathrm{Ph})$, alkaline phosphatase (ALP), parathormone); (ii) intervention safety (eg, as above and serum 25OHD); (iii) covariate documentation (eg, serum total and specific IgE) and (iv) exploratory outcomes (eg, gene expression).

Any abnormal calcium:creatinine value is interpreted as 'clinically significant' or 'not clinically significant' by the blinded Site endocrinologist. If 'clinically significant', a blood aliquot is obtained to measure serum $\mathrm{Ca}, \mathrm{Ph}, \mathrm{ALP}$, or a repeat urine calcium:creatinine is analysed. Further investigation or action for individual patients (including interruption, cessation, or, with prior notification of Site investigator, unblinding of the study drug via pharmacy or by analysis of serum 25OHD) will be selected by the Site endocrinologist, if needed to ensure patient safety. To maintain blinding, serum 25OHD aliquots will be analysed following study completion, unless specifically requested by the site endocrinologist or Data Monitoring Safety Board due to patient safety concerns.

In addition, dietary and supplemental vitamin D intake is systematically monitored throughout the trial to ensure that excess intake is not occurring.

\section{Outcomes}

Primary outcome

The primary outcome, the number of asthma exacerbations requiring OCS per child (ie, within-patient) during the 7-month follow-up, is ascertained by parental report at each contact, and confirmed after the final visit $(7 \pm 0.5$ months) via medical and pharmacy records, with parental consent obtained at enrolment. The success of this strategy has been demonstrated in previous trials, ${ }^{8} 4041$ irrespective of attrition. OCS use is assumed when a patient was administered or prescribed $\geq 1$ OCS dose for an asthma or asthma-like event.

\section{Secondary outcomes}

The mean number of URTIs will be quantified via PCR analysis of nasal swabs, collected by parents during an exacerbation. The $A D Y C^{71}$ is used to ascertain the severity (sum of the daily score) and duration (cumulative number of days with symptoms) of symptoms, and the intensity of rescue $\beta_{2}$-agonist use (cumulative daily number of puffs), during an exacerbation. The ECAP questionnaire ${ }^{72}$ will capture parental functional status during an exacerbation. The mean number of ED visits for asthma is captured via the parent-completed summary-of-event form and confirmed by medical records. Any step-up or step-down in ICS therapy is captured via physician questionnaire at each visit. The intervention cost will be determined by documenting: (i) family expenses during an exacerbation (ie, medications, ED visits, hospitalisations, work absenteeism and out-of-pocket expenses), captured via parent report at each contact; (ii) healthcare cost (ie, ED visits, hospitalisations, medications and physician visits) and (iii) intervention cost, determined through drug manufacturer, nursing time and facility resources.

\section{Safety outcomes}

The primary safety outcome, a 'clinically significant' hypercalciuria event, is based on the site endocrinologist's clinical interpretation of urinary calcium:creatinine results exceeding pre-established laboratory standards. ${ }^{74}$ Similarly, a 'clinically significant' hypercalcaemia event is based on the site endocrinologist's clinical interpretation of serum calcium results exceeding pre-established laboratory standards. Elevated serum 25OHD is defined as a value $>250 \mathrm{nmol} / \mathrm{L},{ }^{64}$ measured at the CHUSJ Central Laboratory after study completion to maintain blinding. Serious and non-serious AHEs will be recorded at each contact in accordance with guidelines adopted by Health Canada. ${ }^{75}$

\section{Exploratory outcomes}

Several outcomes pertaining to immune, infection, inflammation, bone and genetic markers (including changes in gene expression) and lung function will be documented.

\section{Data management and monitoring}

The Applied Clinical Research Unit at CHUSJ will oversee randomisation, data management, progress monitoring and all analyses, including those for the Data Safety Monitoring Board (DSMB). REDCap ${ }^{66}$ will be used for online data entry and management. A combination of remote monitoring activities and routine monitoring visits are conducted to ensure that each Site adheres to the study protocol, Good Clinical Practice guidelines and data collection completeness.

\section{Sample size calculation}

Based on our previous data, ${ }^{8}$ the number of OCS per child is well approximated by a Poisson distribution. The mean number of events per interval $(\lambda)$ was conservatively estimated at $0.55 \mathrm{OCS} /$ child in our placebo arm (based on prorated event rates in preschoolers receiving daily/ pre-emptive ICS in previous trials) ${ }^{8-10}$ A sample size of 400 children per arm will provide $80 \%$ power with a twotailed alpha of $5 \%$ to detect a $25 \%$ relative reduction in the mean number of events (ie, $\lambda=0.55$ in the placebo, vs 
$\lambda=0.4125$ in the intervention, group). This is a conservative estimate, given the observed rate ratio of 0.64 (95\% CI 0.46 to 0.90 ) in a 2016 meta-analysis. ${ }^{13}$ Allowing for $10 \%$ attrition, we aim to recruit 865 children (432/arm).

\section{Statistical analysis \\ Primary outcome}

An intention-to-treat analysis will be carried out with all randomised children by treatment group. The primary outcome will be analysed using a Poisson regression model (including parameters for site stratification) to compute the rate ratio (incidence) for comparing the mean (within-patient) number of OCS per child during the 7-month follow-up, with over-dispersion and offset variable for variations in person-time. A formal interim analysis will be performed when approximately $50 \%$ have been enrolled and completed follow-up. The DSMB will review the interim safety and efficacy data. Interim and final analyses will be adjusted to maintain an overall type I error rate, based on Lan and DeMet's implementation of the O'Brien-Fleming grouped sequential stopping boundary. This implementation will permit early stopping only for strong positive or negative effects and maintains nearly all the nominal power for the final analysis. Seven subgroup analyses are planned on baseline: serum 25OHD ( $<75 \mathrm{vs} \geq 75 \mathrm{nmol} / \mathrm{L}$ ), ICS therapy (pre-emptive vs daily), asthma phenotype (viral-induced vs multitrigger), sex (male/female), atopy (specific multiallergen $\operatorname{IgE} \leq 0.35$ or $>0.35 \mathrm{kUa} / \mathrm{L}),{ }^{68} \mathrm{BMI}$ (Z-score $\leq 2 \mathrm{vs}>2 \mathrm{SD}$ ) ${ }^{76}$ and skin colour (five categories). ${ }^{67}$

\section{Secondary and safety outcomes}

The mean number of laboratory-confirmed viral URTIs will be compared between groups using a negative binomial model that includes a shape parameter and site stratification. Symptom severity during an exacerbation will be compared between groups using a generalised linear regression model, adjusting for event clustering in individuals with an offset variable for variations in person-time, if relevant. Similar analyses will be carried out for symptom duration, intensity of rescue $\beta_{2}$-agonist use, and parental functional status, during an exacerbation. The MantelHaenszel method, stratified by site, will serve to compare categorical outcomes, including the number of children with $\geq 1$ episode of clinically significant hypercalciuria, hypercalcaemia, elevated serum 25OHD, and AHEs; and Poisson regression to compare the mean number of ED visits. No adjustment for multiple outcomes is planned.

The cost-effectiveness of the intervention will be evaluated using standard trial-based economic evaluation methods, employing a nested loop of imputation of missing values (due to attrition), regression (to adjust for baseline variables) and bootstrapping (to estimate uncertainty). ${ }^{77}$ The main outcome of the economic evaluation will be the incremental costs per exacerbation avoided. The main analysis will adopt a public health payer perspective, where direct medical costs will be considered. A secondary analysis, adopting a societal perspective, will evaluate loss of school time and parental/caregiver productivity loss.

\section{Patient and public involvement}

Our patient-engagement initiative ensured parentspartners involvement in the study design and conduct. Consultation with parents of preschoolers with asthma contributed to our primary outcome selection, to approving our intervention and secondary outcomes (eg, disease burden), to enhancing procedures to optimise feasibility and implementation (using electronic reminders and online vs paper completion of questionnaires) and to maximise recruitment, protocol adherence and retention. Parents-partners involved in this trial, and those involved in the Canadian Respiratory Research Network patient platform, will contribute to the dissemination plan to ensure that results are communicated to as many families of preschoolers with asthma as possible.

\section{Ethics and safety considerations}

This study has been independently approved by the CHUSJ Research Ethics Board (REB), serving as the multicentre REB in Quebec; the Children's Hospital of Eastern Ontario REB, serving as the multicentre REB in Ontario and the local REB of all participating institutions (online supplementary file 2), all of which abide by the principles of the Helsinki Declaration. A non-objection letter from Health Canada has been obtained to use highdose vitamin $\mathrm{D}$ and placebo in the context of this study (\#228409). Written informed consent for study participation and biobanking specimens for ancillary studies is obtained from parents of all participants, with the knowledge that participation is voluntary and can be withdrawn at any time with no effect on their current/future medical care (online supplementary file 3). Care will be provided to those who suffer harm from trial participation. All protocol amendments will be submitted to Health Canada, investigators and REBs; if these changes imply a revision of consent forms, ongoing trial participants will be informed of new modifications to provide informed consent.

All information obtained during the study will be kept confidential according to law. Data will be collected directly into electronic Case Report Forms or transcribed from paper versions, as approved by Health Canada. Data safety and confidentiality will be upheld at all data collection stages via assignment of a unique research code to each participant, with data and samples kept under lock and key, electronic password protection and access restricted to study personnel. For medical safety reasons, a copy of the consent form will be included in the participant's medical record, where allowable by the site institution. The principal investigator at each site will be responsible for secure data storage for 25 years and for biobanked specimens as long as adequate management and safeguards can be ensured. The Central site, CHUSJ, will store only coded data. Samples collected during the study will be labelled 
with the unique research code, prior to transfer and storage at the CHUSJ biobank, with access restricted to authorised personnel.

There are two, though extremely low, theoretical risks with vitamin D supplementation in Canadian children: hypocalcaemia, secondary to a rapid increase in bone remodelling at baseline in the condition where the participant presents with an undiagnosed active rickets; and hypercalcaemia/calciuria, due to enhancing effects of vitamin D on intestinal absorption of calcium. Our trial design has incorporated strategies to mitigate risk. We are excluding at enrolment patients at high risk of vitamin $\mathrm{D}$ deficiency and are routinely evaluating calcium and vitamin $\mathrm{D}$ dietary intake, both to exclude the rare child with rickets (Canadian annual incidence $1.6-2.9 / 100000$ children), ${ }^{78}$ in whom vitamin $\mathrm{D}$, without calcium, supplementation could lead to hypocalcaemia. In the first hypothetical case of rickets, ALP levels should also be elevated and be a second flag to exclude this child from the study. Any toxicity in vitamin $\mathrm{D}$ is mediated via elevated serum calcium (with normal or elevated serum phosphorous and normal or low ALP); if serum calcium is normal, there is no clinical risk of vitamin $\mathrm{D}$ toxicity to the individual. To minimise the risk of hypercalcaemia (which could signal vitamin D intoxication), we have selected a bolus dose routinely recommended in France, ${ }^{62}$ with no documented adverse effects, ${ }^{45} 4750$ are excluding children with recent excessive vitamin D supplementation, are systematically screening urine calcium:creatinine (the most sensitive marker of elevated serum calcium) and serum biomarkers (ALP, calcium and phosphorous) at randomisation, with urine screening periodically throughout the trial, and implemented a process whereby all abnormal values are reviewed by the Site endocrinologist for clinical interpretation and management where indicated. Finally, as we expect a fair proportion of enrolled children to have vitamin D insufficiency (ie, $25-75 \mathrm{nmol} / \mathrm{L}$ ) at study entry, all participants will be advised at the end of the study if their usual (dietary and supplemental) vitamin D intake was below recommendations, and provided general dietary advice to meet the Canadian recommended daily intake. $^{33}$

Serious adverse drug reactions will be reported to the Therapeutic Products Directorate (Health Canada) where they are 'unexpected' and the investigator deems there is a reasonable suspected causal relationship to the treatment, or, they are 'expected' and there is an increase in the incidence or the severity is of clinical importance. ${ }^{75}$

An independent DSMB, comprised of relevant experts (paediatric respiratory medicine, paediatric endocrinology, biostatistics and vitamin D) has been established, with terms of reference; it periodically reviews the occurrence of any 'clinically significant' abnormal hypercalciuria/calcaemia results and the number and distribution of AHEs, with notification of serious unexpected AHEs provided within 48 hours of their reporting.

\section{Implementation and dissemination}

This trial employs pragmatic patient selection without serum $25 \mathrm{OHD}$ prescreening and intervention to maximise subsequent implementation into practice. If effective in reducing short-term morbidity, this approach would be readily implementable and could markedly influence asthma management in high-morbidity preschoolers.

No participant identifiers will be used in the dissemination of this research. Results will be disseminated to the medical community via national/international conferences and publications in peer-reviewed journals, and to families of preschoolers with asthma by involving parents-partners in the disseminating plan.

\section{Author affiliations}

${ }^{1}$ Priority Research Centre Grow Up Well, School of Medicine \& Public Health, Faculty of Health, The University of Newcastle, Callaghan, New South Wales, Australia ${ }^{2}$ Department of Social \& Preventive Medicine, University of Montreal, Montreal, Québec, Canada

${ }^{3}$ Clinical Research and Knowledge Transfer Unit on Childhood Asthma, Research Centre, CHU Sainte-Justine, Montreal, Québec, Canada

${ }^{4}$ Department of Pediatrics, University of Montreal, Montreal, Québec, Canada

${ }^{5}$ Pediatric Endocrinology Service, CHU Sainte-Justine, Montreal, Québec, Canada

${ }^{6}$ Department of Nutrition, Faculty of Medicine, University of Montreal, Montreal,

Québec, Canada

${ }^{7}$ Research Centre, CHU Sainte-Justine, Montreal, Québec, Canada

${ }^{8}$ School of Public Health, University of Montreal, Montreal, Québec, Canada

${ }^{9}$ Department of Physiology, McGill University, Montreal, Québec, Canada

${ }^{10}$ Faculty of Pharmaceutical Sciences, The University of British Columbia, Vancouver, British Columbia, Canada

${ }^{11}$ Euro-Pharm International Canada Inc, Montreal, Québec, Canada

${ }^{12}$ Department of Pediatrics, Montreal Children's Hospital, Montreal, Québec, Canada

${ }^{13}$ Department of Pediatrics, Schulich School of Medicine and Dentistry, University of Western Ontario, London, Ontario, Canada

${ }^{14}$ Department of Pediatrics, Centre Mère-Enfant du CHU de Québec, Quebec City,

Quebec, Canada

${ }^{15}$ Division of Allergy and Immunology, Department of Pediatrics, Université de Sherbrooke, Sherbrooke, Quebec, Canada

${ }^{16}$ Department of Pediatrics, British Columbia Children's Hospital, Vancouver, British Columbia, Canada

${ }^{17}$ Department of Pediatrics, Children's Hospital of Eastern Ontario, Ottawa, Ontario, Canada

\section{Twitter Megan E Jensen @DrMeganEJensen}

Contributors FMD designed the study protocol, secured funding and oversees the project. MEJ wrote the first manuscript draft and, along with GM, was involved in the study design and dietary assessment. NA is overseeing the safety assessment. $\mathrm{BM}$ is responsible for the randomisation, data management and statistical analysis. MS is responsible for the economic analysis. JHW is responsible for the gene expression analysis. AK is responsible for the development and provision of the placebo and high-dose vitamin D supplement. All other coauthors (SMT, RA, DEB, $\mathrm{PD}, \mathrm{CL}, \mathrm{CY}$ and $\mathrm{DR}$ ) participated in study design, approved the study protocol and manuscript, and are responsible for conducting the study at their respective sites Authorship eligibility on resulting manuscripts will follow standard guidelines.

Funding This project is funded by a grant awarded through a peer-reviewed process by the Canadian Institutes of Health Research, 160 Elgin Street, Ottawa, ON, K1A 0W9, Canada (grant number \#153252).

Competing interests By partnership agreement, Euro-Pharm International Canada Inc. (Montreal, QC Canada), and their representative (Ali Khamessan) donated all vitamin $D$ study preparations. Dr Khamessan is responsible for the formulation of the placebo and high dose vitamin D preparation; however, neither Dr Khamessan, nor Euro-Pharm International Canada Inc., had any input into other aspects of study design, and will have no involvement in analysis or interpretation of results. The authors have no competing interests.

Patient consent for publication Not required. 
Provenance and peer review Not commissioned; externally peer reviewed.

Open access This is an open access article distributed in accordance with the Creative Commons Attribution Non Commercial (CC BY-NC 4.0) license, which permits others to distribute, remix, adapt, build upon this work non-commercially, and license their derivative works on different terms, provided the original work is properly cited, appropriate credit is given, any changes made indicated, and the use is non-commercial. See: http://creativecommons.org/licenses/by-nc/4.0/.

ORCID iD

Geneviève Mailhot http://orcid.org/0000-0002-2673-6492

\section{REFERENCES}

1 Rosychuk RJ, Voaklander DC, Klassen TP, et al. Asthma presentations by children to emergency departments in a Canadian Province: a population-based study. Pediatr Pulmonol 2010;45:985-92.

2 Lougheed MD, Garvey N, Chapman KR, et al. The Ontario asthma regional variation study: emergency department visit rates and the relation to hospitalization rates. Chest 2006;129:909-17.

3 Khetsuriani N, Kazerouni NN, Erdman DD, et al. Prevalence of viral respiratory tract infections in children with asthma. J Allergy Clin Immunol 2007;119:314-21.

4 Xepapadaki P, Papadopoulos NG. Childhood asthma and infection: virus-induced exacerbations as determinants and modifiers. Eur Respir J 2010;36:438-45.

5 Miller EK, Edwards KM, Weinberg GA, et al. A novel group of rhinoviruses is associated with asthma hospitalizations. J Allergy Clin Immunol 2009;123:98-104.

6 Ducharme FM, Zemek R, Chauhan BF, et al. Factors associated with failure of emergency department management in children with acute moderate or severe asthma: a prospective, multicentre, cohort study. Lancet Respir Med 2016;4:990-8.

7 Ducharme FM, Tse SM, Chauhan B. Diagnosis, management, and prognosis of preschool wheeze. Lancet 2014;383:1593-604.

8 Ducharme FM, Lemire C, Noya FJD, et al. Preemptive use of highdose fluticasone for virus-induced wheezing in young children. $N$ Engl J Med 2009;360:339-53.

9 Bacharier LB, Phillips BR, Zeiger RS, et al. Episodic use of an inhaled corticosteroid or leukotriene receptor antagonist in preschool children with moderate-to-severe intermittent wheezing. J Allergy Clin Immunol 2008;122:1127-35.

10 Zeiger RS, Mauger D, Bacharier LB, et al. Daily or intermittent budesonide in preschool children with recurrent wheezing. $N$ Engl J Med 2011;365:1990-2001.

11 Vrijlandt EJLE, El Azzi G, Vandewalker M, et al. Safety and efficacy of tiotropium in children aged 1-5 years with persistent asthmatic symptoms: a randomised, double-blind, placebo-controlled trial. Lancet Respir Med 2018;6:127-37.

12 Holt PG, Sly PD. Viral infections and atopy in asthma pathogenesis: new rationales for asthma prevention and treatment. Nat Med 2012;18:726-35

13 Martineau AR, Cates CJ, Urashima M, et al. Vitamin D for the management of asthma. Cochrane Database Syst Rev 2016;9.

14 Alansari K, Davidson BL, Yousef KI, et al. Rapid vs Maintenance Vitamin D Supplementation in Deficient Children With Asthma to Prevent Exacerbations. Chest 2017;152:527-36.

15 Kerley CP, Hutchinson K, Cormican L, et al. Vitamin $\mathrm{D}_{3}$ for uncontrolled childhood asthma: A pilot study. Pediatr Allergy Immunol 2016;27:404-12.

16 Paul G, Brehm JM, Alcorn JF, et al. Vitamin D and asthma. Am J Respir Crit Care Med 2012;185:124-32.

17 Bergman P, Lindh Åsa U., Björkhem-Bergman L, et al. Vitamin D and respiratory tract infections: a systematic review and meta-analysis of randomized controlled trials. PLoS One 2013;8:e65835.

18 Charan J, Goyal J, Saxena D, et al. Vitamin D for prevention of respiratory tract infections: a systematic review and meta-analysis. $J$ Pharmacol Pharmacother 2012;3:300-3.

19 Xiao L, Xing C, Yang Z, et al. Vitamin D supplementation for the prevention of childhood acute respiratory infections: a systematic review of randomised controlled trials. Br J Nutr 2015;114:1026-34.

20 Urashima M, Segawa T, Okazaki M, et al. Randomized trial of vitamin D supplementation to prevent seasonal influenza A in schoolchildren. Am J Clin Nutr 2010;91:1255-60.

21 Hughes DA, Norton R. Vitamin D and respiratory health. Clin Exp Immunol 2009;158:20-5.

22 Herr C, Greulich T, Koczulla RA, et al. The role of vitamin D in pulmonary disease: COPD, asthma, infection, and cancer. Respir Res 2011;12:31.
23 Finklea JD, Grossmann RE, Tangpricha V. Vitamin D and chronic lung disease: a review of molecular mechanisms and clinical studies. Adv Nutr 2011;2:244-53.

24 Dimitrov V, Salehi-Tabar R, An B-S, et al. Non-Classical mechanisms of transcriptional regulation by the vitamin $D$ receptor: insights into calcium homeostasis, immune system regulation and cancer chemoprevention. J Steroid Biochem Mol Biol 2014;144:74-80.

25 Carlberg C, Seuter S, de Mello VDF, et al. Primary vitamin D target genes allow a categorization of possible benefits of vitamin D3 supplementation. PLoS One 2013;8:e71042.

26 Bossé Y, Maghni K, Hudson TJ. $1 \alpha, 25$-Dihydroxy-vitamin $\mathrm{D}_{3}$ stimulation of bronchial smooth muscle cells induces autocrine, contractility, and remodeling processes. Physiol Genomics 2007;29:161-8.

27 Chinellato I, Piazza M, Sandri M, et al. Vitamin D serum levels and markers of asthma control in Italian children. J Pediatr 2011;158:437-41.

28 Strachan DP, Griffiths JM, Johnston ID, et al. Ventilatory function in British adults after asthma or wheezing illness at ages 0-35. Am J Respir Crit Care Med 1996;154:1629-35.

29 Gupta A, Sjoukes A, Richards D, et al. Relationship between serum vitamin $D$, disease severity, and airway remodeling in children with asthma. Am J Respir Crit Care Med 2011;184:1342-9.

30 Goleva E, Searing DA, Jackson LP, et al. Steroid requirements and immune associations with vitamin $D$ are stronger in children than adults with asthma. J Allergy Clin Immunol 2012;129:1243-51.

31 Searing DA, Zhang Y, Murphy JR, et al. Decreased serum vitamin $D$ levels in children with asthma are associated with increased corticosteroid use. J Allergy Clin Immunol 2010;125:995-1000.

32 Brehm JM, Celedón JC, Soto-Quiros ME, et al. Serum vitamin D levels and markers of severity of childhood asthma in Costa Rica. Am J Respir Crit Care Med 2009;179:765-71.

33 Godel JC. Vitamin D supplementation: recommendations for Canadian mothers and infants. Paediatr Child Health 2007;12:583-9.

34 Rosen CJ, Adams JS, Bikle DD, et al. The nonskeletal effects of vitamin D: an endocrine Society scientific statement. Endocr Rev 2012;33:456-92.

35 Schwalfenberg GK. A review of the critical role of vitamin D in the functioning of the immune system and the clinical implications of vitamin D deficiency. Mol Nutr Food Res 2011;55:96-108.

36 Cassim R, Russell MA, Lodge CJ, et al. The role of circulating 25 hydroxyvitamin $D$ in asthma: a systematic review. Allergy 2015;70:339-54.

37 El Hayek J, Pham TT, Finch S, et al. Vitamin D status in Montréal preschoolers is satisfactory despite low vitamin D intake. J Nutr 2013;143:154-60.

38 Bener A, Ehlayel MS, Tulic MK, et al. Vitamin D deficiency as a strong predictor of asthma in children. Int Arch Allergy Immunol 2012;157:168-75.

39 Freishtat RJ, lqbal SF, Pillai DK, et al. High prevalence of vitamin D deficiency among inner-city African American youth with asthma in Washington, DC. J Pediatr 2010;156:948-52.

40 Ducharme FM, Jensen M, Mailhot G, et al. Impact of two oral doses of 100,000 IU of vitamin D3 in preschoolers with viral-induced asthma: a pilot randomised controlled trial. Trials 2019;20:138.

41 Jensen ME, Mailhot G, Alos N, et al. Vitamin D intervention in preschoolers with viral-induced asthma (Diva): a pilot randomised controlled trial. Trials 2016;17:353.

42 Maguire JL, Birken CS, Khovratovich M, et al. Modifiable determinants of serum 25-hydroxyvitamin D status in early childhood: opportunities for prevention. JAMA pediatrics 2013;167:230-5.

43 Johnston NW, Johnston SL, Duncan JM, et al. The September epidemic of asthma exacerbations in children: a search for etiology. $J$ Allergy Clin Immunol 2005;115:132-8.

44 Martineau AR, Jolliffe DA, Hooper RL, et al. Vitamin D supplementation to prevent acute respiratory tract infections: systematic review and meta-analysis of individual participant data. BMJ 2017;356.

45 Manaseki-Holland S, Maroof Z, Bruce J, et al. Effect on the incidence of pneumonia of vitamin D supplementation by quarterly bolus dose to infants in Kabul: a randomised controlled superiority trial. Lancet 2012;379:1419-27.

46 Manaseki-Holland S, Qader G, Isaq Masher M, et al. Effects of vitamin D supplementation to children diagnosed with pneumonia in Kabul: a randomised controlled trial. Trop Med Int Health 2010;15:1148-55.

47 Zeghoud F, Ben-Mekhbi H, Djeghri N, et al. Vitamin D prophylaxis during infancy: comparison of the long-term effects of three intermittent doses $(15,5$, or $2.5 \mathrm{Mg})$ on 25 -hydroxyvitamin D concentrations. Am J Clin Nutr 1994;60:393-6. 
48 Marwaha RK, Tandon N, Agarwal N, et al. Impact of two regimens of vitamin D supplementation on calcium - vitamin D - PTH axis of schoolgirls of Delhi. Indian Pediatr 2010;47:761-9.

49 Oliveri B, Cassinelli H, Mautalen C, et al. Vitamin D prophylaxis in children with a single dose of 150000 IU of vitamin D. Eur J Clin Nutr 1996;50:807-10.

50 Tau C, Ciriani V, Scaiola E, et al. Twice single doses of 100,000 U of vitamin $D$ in winter is adequate and safe for prevention of vitamin D deficiency in healthy children from Ushuaia, Tierra del Fuego, Argentina. J Steroid Biochem Mol Biol 2007;103:651-4.

51 Osunkwo I, Ziegler TR, Alvarez J, et al. High dose vitamin D therapy for chronic pain in children and adolescents with sickle cell disease: results of a randomized double blind pilot study. $\mathrm{Br} J$ Haematol 2012;159:211-5.

52 Pappa HM, Mitchell PD, Jiang H, et al. Treatment of vitamin D insufficiency in children and adolescents with inflammatory bowel disease: a randomized clinical trial comparing three regimens. J Clin Endocrinol Metab 2012;97:2134-42.

53 Ward KA, Das G, Roberts SA, et al. A randomized, controlled trial of vitamin D supplementation upon musculoskeletal health in postmenarchal females. J Clin Endocrinol Metab 2010;95:4643-51.

54 Mallet E, Philippe F, Castanet M, et al. Administration of a single winter oral dose of 200,000 IU of vitamin D3 in adolescents in Normandy: evaluation of the safety and vitamin D status obtained. Arch Pédiatr 2010;17:1042-6.

55 Martineau AR, Nanzer AM, Satkunam KR, et al. Influence of a single oral dose of vitamin $\mathrm{D}(2)$ on serum 25-hydroxyvitamin $\mathrm{D}$ concentrations in tuberculosis patients. Int J Tuberc Lung Dis 2009;13:119-25.

56 Grossmann RE, Zughaier SM, Kumari M, et al. Pilot study of vitamin D supplementation in adults with cystic fibrosis pulmonary exacerbation. Dermatoendocrinol 2012;4:191-7.

57 Havens PL, Mulligan K, Hazra R, et al. Serum 25-hydroxyvitamin D response to vitamin D3 supplementation 50,000 IU monthly in youth with HIV-1 infection. J Clin Endocrinol Metab 2012;97:4004-13.

58 Leventis P, Kiely PDW. The tolerability and biochemical effects of high-dose bolus vitamin D2 and D3 supplementation in patients with vitamin D insufficiency. Scand J Rheumatol 2009;38:149-53.

59 Sainaghi PP, Bellan M, Nerviani A, et al. Superiority of a high loading dose of cholecalciferol to correct hypovitaminosis $D$ in patients with Inflammatory/Autoimmune rheumatic diseases. J Rheumatol 2013;40:166-72.

60 Stoll D, Dudler J, Lamy O, et al. Can one or two high doses of oral vitamin D3 correct insufficiency in a non-supplemented rheumatologic population? Osteoporosis International 2013;24:495-500.

61 Wejse C, Gomes VF, Rabna P, et al. Vitamin D as supplementary treatment for tuberculosis: a double-blind, randomized, placebocontrolled trial. Am J Respir Crit Care Med 2009;179:843-50.

62 Vidailhet M, Mallet E, Bocquet A, et al. Vitamin D: still a topical matter in children and adolescents. A position paper by the Committee on nutrition of the French Society of paediatrics. Archives de Pédiatrie 2012;19:316-28.

63 Ducharme FM, Dell SD, Radhakrishnan D, et al. Diagnosis and management of asthma in preschoolers: a Canadian thoracic Society and Canadian paediatric Society position paper. Can Respir J 2015;22:135-43.

64 Jones G. Pharmacokinetics of vitamin D toxicity. Am J Clin Nutr 2008;88:582S-6.

65 IOM. Institute of medicine). dietary reference intakes for calcium and vitamin D. Washington DC: The National Academies Press, 2011.

66 Harris PA, Taylor R, Thielke R, et al. Research electronic data capture (REDCap)-A metadata-driven methodology and workflow process for providing translational research informatics support. J Biomed Inform 2009;42:377-81.

67 Fitzpatrick TB. The validity and practicality of sun-reactive skin types I through VI. Arch Dermatol 1988;124:869-71.

68 Szefler SJ, Wenzel S, Brown R, et al. Asthma outcomes: biomarkers. Journal of Allergy and Clinical Immunology 2012;129:S9-23.

69 Raymond F, Carbonneau J, Boucher N, et al. Comparison of automated microarray detection with real-time PCR assays for detection of respiratory viruses in specimens obtained from children. J Clin Microbiol 2009;47:743-50.

70 Ali M, Han S, Gunst CJ, et al. Throat and nasal swabs for molecular detection of respiratory viruses in acute pharyngitis. Virol $J$ 2015;12:178.

71 Ducharme FM, Jensen ME, Mendelson MJ, et al. Asthma Flare-up diary for young children to monitor the severity of exacerbations. $J$ Allergy Clin Immunol 2016;137:744-9.

72 Jensen ME, Mendelson MJ, Desplats E, et al. Caregiver's functional status during a young child's asthma exacerbation: a validated instrument. J Allergy Clin Immunol 2016;137:782-8.

73 Esposito S, Molteni CG, Daleno C, et al. Collection by trained pediatricians or parents of mid-turbinate nasal flocked swabs for the detection of influenza viruses in childhood. Virol $J$ 2010;7:85.

74 Metz MP. Determining urinary calcium/creatinine cut-offs for the paediatric population using published data. Ann Clin Biochem 2006;43:398-401.

75 International Conference on Harmonisation Expert Working Group. Clinical safety data management: definitions and standards for Expedited reporting E2A, 1994

76 Mazahery $H$, von Hurst P. Factors affecting 25-hydroxyvitamin D concentration in response to vitamin D supplementation. Nutrients 2015;7:5111-42.

77 Briggs $\mathrm{AH}$, Wonderling DE, Mooney CZ. Pulling cost-effectiveness analysis up by its bootstraps: a non-parametric approach to confidence interval estimation. Health Econ 1997;6:327-40.

78 Ward LM, Gaboury I, Ladhani M, et al. Vitamin D-deficiency rickets among children in Canada. Can Med Assoc J 2007;177:161-6.

79 Lautatzis M-E, Sharma A, Rodd C. A closer look at rickets and vitamin D deficiency in Manitoba: the tip of the iceberg. Paediatr Child Health 2019;24:179-84. 